\title{
Ran GTPase is an independent prognostic marker in malignant melanoma which promotes tumour cell migration and invasion
}

Somaia Elsheikh ${ }^{*{ }^{* 1}, 2,3}$, llias Kouzoukakis ${ }^{* 3}$, Catherine Fielden ${ }^{3}$, Wei $\mathrm{Li}^{3}$, Shaimaa Lashin $^{2,3}$, Nadia Khair²,Teresa Pereira Raposo ${ }^{3}$, Wakkas Fadhil ${ }^{3}$, Philip Rudland ${ }^{4,5}$, Mohammed Aleskandarany ${ }^{2,3}$, Poulam Patel ${ }^{3}$, Mohamed El-Tanani ${ }^{6,7,8, * *}$, Mohammad Ilyas ${ }^{1,3 * *}$.

1-Cellular Pathology Department, Nottingham University Hospital, UK.

2- School of Medicine, Menoufyia University, Egypt.

3- Division of Cancer and Stem cells, School of Medicine, University of Nottingham, UK.

4 -School of Biological Sciences, Chemical and Translational Biology, University of Edinburgh, UK

5- School of Biological Sciences, University of Liverpool, UK

6- School of Chemistry and Biosciences, University of Bradford.

7- Institute of Cancer Therapeutics, University of Bradford.

8-Pharmacological and Diagnostic Research Centre, Faculty of Pharmacy, Al-Ahliyya Amman University

* Shared first author

** Equal contribution

Corresponding author details:

Dr. Somaia Elsheikh Cellular Pathology Department.

Nottingham University Hospital and University of Nottingham, School of Medicine Queen's Medical Centre

Derby Road

NG7 2UH

Fax :01159704852

Tel: 01159249924 Ext 61169.

Mail: Somaia.elsheikh@nottingham.ac.uk 


\section{Abstract}

\section{Introduction}

Ran GTPase is involved in nucleocytoplasmic shuttling of proteins and is overexpressed in several cancers. The expression of Ran in malignant melanoma (MM) and its functional activity have not been described and were investigated in this study.

\section{Materials and Methods}

The prognostic value of Ran expression was tested in a series of 185 primary cutaneous malignant melanoma (MM) cases using immunohistochemistry (IHC). The functional activity of Ran was investigated in the two melanoma cell lines. Ran expression was knocked down using two small interfering RNAs (siRNAs) and the effect on the expression of the C-Met oncogene, a potential downstream target of Ran, was tested. Functional effects of Ran knockdown on cell motility and cell proliferation were also assessed.

\section{Results}

Positive Ran expression was seen in $12.4 \%$ of MM and was associated with advanced clinical stage and greater Breslow thickness. Positive expression was an independent marker of shorter overall survival $(p=0.023)$. Knockdown of Ran results in decreased expression of C-Met and the downstream C-met signalling targets ERK1/2. There was

a significant reduction in cell migration $(p<0.001)$ and cell invasion $(p<0.001)$. C-Met knockdown decreased the expression of Ran through MAPK and PI3K-AKT in A375 cell line, inhibited the cell viability and migration of both A375 and G361 melanoma cell lines whilst invasion was enhanced.

\section{Conclusion}

Ran is a poor prognostic marker in cutaneous malignant melanoma. It up-regulates expression of the oncogene C-Met and, possibly through this, it promotes cell motility which may in turn promote metastasis.

\section{Keywords}

Melanoma, Ran, C-Met, immunohistochemistry, prognosis, cell motility 


\section{Introduction}

Malignant Melanoma (MM) is an aggressive skin cancer and is notorious for its high metastatic potential (1). Metastatic MM is characterised by high mortality rates and resistance to chemotherapeutic agents (1-4). A number of driver genes have been identified (such as BRAF, KIT, NRAS and NF1) which are associated with the initiation and progression of MM (5-8). Nevertheless, the precise molecular mechanisms that drive tumour progression and biological behaviour are unclear.

The Ras superfamily of GTPases controls a remarkable number of diverse cellular functions including signal transduction, nucleocytoplasmic transportation, microtubule assemble and nuclear envelope formation (9-12).

Ran GTPase (Ran) is a low-weight ( $29 \mathrm{kDa})$ member of the Ras superfamily that relies on its acidic carboxyl terminus to perform its physiological roles (13). Its principal function is to regulate the transport of macromolecules, including RNA and proteins, between nucleus and cytoplasm (14). Ran overexpression has been reported in several different cancer types, including renal, breast, ovarian, lung and colon (15-18). Overexpression is seen at both mRNA and protein levels and positively correlates with aggressive features such as increased cell proliferation, migration and invasion (19). Recently, we have shown, in lung and breast cancer, that Ran expression may promote cancer cell survival through up-regulation of the C-Met signalling pathway (20, $21)$. In these tumours, Ran may be a potential therapeutic target $(22,23)$.

There is little information on Ran expression and function in MM. Given the importance of other members of the Ras superfamily (such as NRAS) and associated molecules (such as BRAF which is a downstream signal transduction target of the KRAS), we hypothesised that Ran may play a role in the development and progression of MM.. We have shown that silencing Ran expression induces more apoptosis in activated KRas mutant cells compared to their isogenic K-Ras wildtype counterparts (20). We have also shown that C-MET is mediating resistance to BRAF inhibitors in BRAFV600E mutant melanoma and established a dominant role for the HGF/MET axis.(24). Therefore, we hypothesised that Ran may also play a role in the development and progression of MM.

In the present study, we first investigated the value of Ran protein expression as a prognostic biomarker. A series of 185 cases of primary cutaneous MM was tested by immunohistochemistry (IHC) and the significance of Ran expression on overall 
survival as well as lymph node-metastasis-free survival in patients with MM was evaluated. We next tested the functional activity of Ran in MM, in particular its effect on expression of the oncogene C-Met protein and its regulation of biological processes such as cell motility and proliferation.

\section{Materials and Methods}

\section{Patient data}

Cases were selected from patients consecutively diagnosed with MM between June 2008 and February 2017 at Nottingham University Hospitals, UK, based on the availability of sufficient melanoma tissue in formalin-fixed paraffin-embedded (FFPE) blocks for tissue microarray construction. This created a cohort of 228 cases. Further details on the patient cohort and the tissue microarray construction are given in the Supplementary Data and Table 1S.

\section{Immunohistochemistry}

Immunohistochemistry (IHC) was performed using Ran monoclonal antibody (Abcam, ab4781) as previously described (25) and full details are given in the supplementary Data. Sections were scored independently by CF and SE under the microscope using the $\mathrm{H}$-score method as described previously (26). Briefly, the intensity of Ran cytoplasmic expression was categorised as 0 (no staining), 1 (weak staining), 2 (moderate staining) or 3 (strong staining). The percentage of melanoma cells in each category was multiplied by the value of the category resulting in an $\mathrm{H}$-score of 0-300. X-tile software version 3.6.1 (Yale University, USA) was used to generate an outcome dependent cut-off point of RAN H-score as a threshold to dichotomise the tumours into positive (high expression) and negative (low expression) which happened to be equivalent to the median $\mathrm{H}$-score.

\section{Cell culture and Ran gene knockdown}

Melanoma cell lines A375 and G361 were obtained from the European Collection of Authenticated Cell Cultures (ECACC) and confirmed by genotyping. Cells were cultured in DMEM (Life Technologies, UK) supplemented with 10\% v/v foetal bovine serum (Fisher Scientific, UK) and 4mM L-Glutamine (Sigma Aldrich, UK). Expression of Ran was knocked down using two siRNAs targeted to different parts of the Ran mRNA. Transfection with siRNA targeted to the non-mammalian gene luciferase was used as a control. Full details of cell transfection are shown in in supplementary data.

\section{Cell migration and invasion assays}


Cell migration and invasion were assessed in transwells with $8 \mu \mathrm{m}$-pore polycarbonate membranes (Costar) as previously described (20).

Cell invasion was measured in the same way, except that prior to cell seeding the upper chamber was prepared by coating the filter with $100 \mu$ l Matrigel at $0.3 \mathrm{mg} / \mathrm{mL}$ (BD Biosciences) and the cells attached to the lower surface were fixed, stained and counted. Further details including wound healing assay is provided in the supplementary data.

\section{Cell viability assay}

A total of $1 \times 10^{4}$ cells $/ 100 \mu \mathrm{L}$ was seeded per well of a 96 -well culture plate. Twelve replicates were performed for each cell line and experiments were repeated at least twice independently. Detailed protocol is provided in supplementary data.

\section{Statistical analysis}

Statistical analysis was carried out using GraphPad Prism software for functional studies and SPSS version 24. Further details are given in the Supplementary Data.

\section{Results}

\section{Ran expression in MM by Immunohistochemistry}

Twenty cases of MM were evaluated as whole tissue sections in order to evaluate patterns of staining as a preliminary step to test for the reliability of TMA in assessing Ran protein expression. Sixty-three cases from the initial cohort of 248 were excluded due to tissue loss or insufficient melanoma cells for scoring.

Melanoma cells show cytoplasmic staining with occasional membrane accentuation (Figure 1). Ran staining was semi-quantitatively assessed using the $\mathrm{H}$-score. All cases were assessed by two observers with a substantial inter-observer agreement (kappa coefficient $=0.7$ ).

The $\mathrm{H}$-scores ranged from 0 to 245 with a median $\mathrm{H}$-score of 102 . This was the threshold to dichotomise cases as Ran positive (high expression) (H-score $>102)$ or Ran negative (absent or low expression) ( $\mathrm{H}$-score $\leq 102)$. At this cut-off point, a total of $23 / 185$ (12.4\%) of the MM cohort was Ran positive.

\section{The association between Ran expression and standard prognostic parameters}

Ran expression had a significant positive association with adverse prognostic parameters (Table, 2S). A higher proportion of cases with Breslow thickness (BT) >2$4 \mathrm{~mm}$ (56.5\%) had positive Ran expression while the 1-2 $\mathrm{mm} \mathrm{BT}$ group had the lowest rate of expression (13\%) $(p=0.019)$. Ran expression also had a significant positive 
association with age $(\mathrm{p}=0.004)$ where a higher proportion of RAN positive cases were $>70$ years old $(p=0.004)$. No significant association was found with patient's gender, melanoma ulceration, melanoma subtype, microsatellites or local recurrence.

\section{Ran expression is an independent marker of poor prognosis in MM}

Kaplan-Meier plots and Log Rank tests were used to assess the association of Ran expression and lymph node metastasis-free survival (LMFS) and overall survival (OS). Results revealed that Ran negative patients have a statistically significant longer LMFS ( $p=0.045)$ (Figure 2A). Positive Ran expression was also associated with significant reduction in OS. The mean OS for Ran negative cases was 98.8. SE \pm 3.5 months versus 67.3 SE \pm 9.3 months for Ran positive cases ( $p=0.001$, Figure 2B).

Multivariate Cox proportional hazards model was used to quantify the effect of each significant prognostic factor, including Ran expression, on overall survival (Table 1). Data showed that there was a significant increased risk of mortality with male gender (Hazard Ratio (HR) 3.3, $p=0.008$ ), old age (HR 3, $p=0.009$ ), BT $\geq 5.01 \mathrm{~mm}$ (HR 6.7, $<0.001$ ), and ulceration (HR 2.9, 0.003). Positive Ran expression was found to be an independent prognostic marker and associated with a 1.9-fold increased risk of mortality $(p<0.038)$.

\section{Ran is a regulator of C-Met/ERK expression in MM cell lines}

Western blot and qPCR showed that Ran is expressed in both A375 and G361 cell lines (Figure 1S). Both siRNAs were able to reduce Ran protein expression although siRAN2 was slightly more efficient than siRAN1 (Figure 2S). Ran knockdown resulted in reduction of CMet expression in both cell lines with either siRNAs (Figure 3). Since ERK1/2 is part of the downstream CMet signalling pathway, levels of these proteins were also tested. Knockdown of Ran expression in both A375 and G361 resulted in reduction of both total ERK 1/2 and Phospho-ERK1/2 levels (Figure 3).

\section{Knockdown of Ran reduces melanoma cell migration and invasion}

Melanoma cell migration was assessed with transwell migration assays. Ran knockdown with either siRAN1 or siRAN2 resulted in significantly reduced cell migration $(p<0.001)$ for each siRNAs in both cell lines (Figure 4A). In the wound healing assay, the A375 cell line showed data consistent with the transwell migration assay, since there was a significant reduction in wound closure following Ran knockdown, as illustrated in Figure $4 \mathrm{C}$ at 24h ( $\operatorname{liRan} 1$ vs siluc $\mathrm{p}=0.0003$, siRan2 vs siLuc $p<0.0001$, Figure 4B). 
Following Ran knockdown, invasiveness was also significantly reduced by both siRNAs in A375 (siRan1 vs siluc $\mathrm{P}=0.0004$, siRan2 vs siLuc $\mathrm{P}<0.0001$ ) and $\mathrm{G} 361$ (siRan1 vs siLuc $P=0.0073$, siRan2 vs siLuc $p<0.0001$, Figure 4D) cells.

Cell viability was measured 24 hours after transfection, using multiple replicates. There was possibly a trend for reduced cell viability upon Ran knockdown but this was not significant ( $\mathrm{P}=0.0512$ ) (Figure 4E and 4F).

\section{C-Met knockdown signalling and functional effects}

As shown in Figure 5A and 5B, C-Met was successfully knocked down by using siRNA in both cell lines during a $72 \mathrm{~h}$ time course, although in the G361 cell line there was a return in expression of $\mathrm{C}-$ Met at $48 \mathrm{~h}$, it was still less expressed than the negative control group.

Figure $5 C$ and $5 D$ illustrate that phosphorylation of both ERK $1 / 2$ and AKT decreased in either cell line after 48h of C-Met knockdown. At 72h, phosphorylated ERK1/2 was increased in both cell lines but that of AKT either remained the same in A375 or increased in G361 cells. Ran expression increases in A375 but not G361 cell lines. The C-Met knockdown in A375 cell line caused the increase of Ran expression at $24 \mathrm{hr}$, but the expression decreased at $48 \mathrm{~h}$ and then increased at $72 \mathrm{hr}$. This result is consistent with the knockdown results that show phosphorylated MAPK and/or phosphorylated AKT were downregulated at $48 \mathrm{hr}$, but then upregulated at $72 \mathrm{hr}$.

Knockdown of C-Met inhibits the cell viability of both cell lines at $48 \mathrm{~h}(\mathrm{p}<0.0001$ for G361 and $p=0.0037$ for A375) as shown in figure $6 A$ and $6 B$.

C-Met knockdown after 48h significantly decreased the migration of the A375 cell line $(p=0.0054)$, but not the $G 361$ cell line $(p=0.1456)$ (Figure $6 C)$. Interestingly, after $72 h$ of transfection, C-Met knockdown enhanced the invasion of the G361 and A375 cell lines, although statistical significance was only achieved in the G361 cells (Figure $6 \mathrm{D})$.

\section{Discussion}

The list of tumours in which Ran GTPase plays an important role is ever-increasing and we have previously shown that it is involved in breast and lung cancers $(15,21$, 27). Herein we report a potential role for Ran in the development and progression of malignant melanoma (MM). This study shows that Ran is differentially expressed in MM tissue from a cohort of 185 patients. Positive expression is seen in $12.4 \%$ of tumours and is significantly associated with features of poor prognosis such as increased Breslow thickness and age. An association with poor OS $(p=0.001)$ is 
observed and multivariate analysis shows that it is an independent prognostic marker with a 1.9-fold increase in the risk of death if the tumour shows high Ran expression. These findings suggest that Ran could be a useful biomarker which would improve prognostication in MM and, given that there are small molecule inhibitors of Ran, it could become a potential therapeutic target in melanoma.

The significant association seen between Ran expression and lymph node metastasisfree survival ( $p=0.001$ ) prompted us to hypothesise that Ran could be involved in the metastatic process. We have previously shown that Ran up-regulates the oncogene C-Met in breast and lung cancer. C-Met is a receptor for motility-inducing Hepatocyte Growth Factor [24] and thus provides a plausible mechanism for Ran affecting metastasis through modulation of cell motility. Knockdown of Ran using two different siRNAs in two different melanoma cell lines resulted in down-regulation of C-Met expression. This was also associated with down-regulation of both total and phosphorylated ERK1/2; this is a downstream signalling target of C-met and implies that Ran-induced changes in C-Met protein result in changes in C-Met function. Since we have observed that Ran knockdown results in a significant reduction of cell motility (both cell migration and cell invasion) we can tentatively infer that this may be mediated through regulation of $\mathrm{C}$-met. Our data contrast with those reported by Caputo et al. who did not find any effect of Ran on melanoma cell motility (28), although they did suggest that Ran may have a role in the regulation of C-Met in MM.

By manipulating C-Met expression, through siRNA knockdown, we have observed inhibition of cell viability and enhancement of cell invasion, as well as decreased migration for A375.This may be influenced by MAPK and/or AKT temporary downregulation, as well as Ran late increase at $72 \mathrm{~h}$. In dedifferentiated liposarcoma, C-Met knockdown has been reported to decrease invasion, migration and tumorigenicity in vitro (29). However, as different methodology and tumour types were employed in our case, the precise mechanisms of C-Met regulation by Ran remain to be determined. Ran could control the flux of C-Met RNA directly across the nuclear membrane or it may regulate C-Met indirectly, by controlling other molecules which affect C-Met expression levels. In this case, as our data suggest, it is possible that the functional effects of Ran on cell migration and invasion are mediated through C-Met. Hence, an assessment of Ran expression may allow stratification of patients into groups to be treated with either anti-Ran or anti-C-Met therapy. 
Whilst our observations on the role of Ran in melanoma are broadly consistent with our data in other tumour types, one discrepancy is the effect of Ran on cell viability. We found that Ran knockdown does not appear to influence cell viability in the melanoma cell lines studied and, whilst this validates our cell migration and invasion assays, it contradicts results from Caputo et al. in MM and also our own observations in other tumour types (28). In this particular case, as our data suggests, it is possible that the functional effects of Ran on cell migration and invasion are mediated through C-Met.

In summary, to the best of our knowledge, this is the first report showing Ran induces cell migration and cell invasion in malignant melanoma. This may be effect mediated through its role as a positive regulator of C-Met expression. Furthermore, this effect is the first report of Ran as an independent marker of poor prognosis that could also be used to stratify patients into Ran/C-Met treatment subgroups. 
Table 1: Multivariate hazard effect of Ran expression and clinicopathological parameters on Overall survival.

\begin{tabular}{|c|c|c|c|c|}
\hline Factor & & $\begin{array}{l}\text { Hazard } \\
\text { Ratio }\end{array}$ & $\begin{array}{c}\text { 95\% Confidence } \\
\text { Interval }\end{array}$ & $\begin{array}{c}\text { p- } \\
\text { value }\end{array}$ \\
\hline \multirow[t]{2}{*}{ Gender } & Male & \multirow[t]{2}{*}{3.334} & \multirow[t]{2}{*}{$1.364-8.149$} & \multirow[t]{2}{*}{0.008} \\
\hline & Female & & & \\
\hline \multirow[t]{2}{*}{ Age } & $<70$ & \multirow[t]{2}{*}{3.056} & \multirow[t]{2}{*}{$1.325-7.048$} & \multirow[t]{2}{*}{0.009} \\
\hline & $>70$ & & & \\
\hline \multirow{2}{*}{$\begin{array}{l}\text { Breslow } \\
\text { thickness }\end{array}$} & $>5.00$ & \multirow[t]{2}{*}{6.748} & \multirow[t]{2}{*}{$2.993-15.211$} & \multirow[t]{2}{*}{$<0.001$} \\
\hline & $\geq 5.01$ & & & \\
\hline \multirow[t]{2}{*}{ Ulceration } & Absent & \multirow[t]{2}{*}{2.924} & \multirow[t]{2}{*}{$1.440-5.934$} & \multirow[t]{2}{*}{0.003} \\
\hline & Present & & & \\
\hline \multirow[t]{2}{*}{ Mitosis } & $<4 / \mathrm{mm}^{2}$ & \multirow[b]{2}{*}{2.093} & \multirow[t]{2}{*}{$1.031-4.249$} & \multirow[t]{2}{*}{0.041} \\
\hline & $>4 / \mathrm{mm}^{2}$ & & & \\
\hline \multirow[t]{2}{*}{ Ran Status } & Negative & \multirow[b]{2}{*}{1.969} & \multirow[t]{2}{*}{$1.040-3.727$} & \multirow[t]{2}{*}{0.038} \\
\hline & Positive & & & \\
\hline
\end{tabular}




\section{Conflict of interest statement}

The authors declare that there is no conflict of interest regarding the publication of this article

\section{Acknowledgements}

This work was partially supported by the University of Nottingham and the Supreme Council of Universities, Egypt. 


\section{References}

1. Arozarena I, Wellbrock C. Targeting invasive properties of melanoma cells. FEBS J. 2017;284(14):2148-62.

2. Cherobin A, Wainstein AJA, Colosimo EA, Goulart EMA, Bittencourt FV. Prognostic factors for metastasis in cutaneous melanoma. An Bras Dermatol. 2018;93(1):19-26.

3. Messeguer F, Agusti-Mejias A, Traves V, Requena C, Alegre V, Guillen C, et al. Mitotic rate and subcutaneous involvement are prognostic factors for survival after recurrence in patients with only locoregional skin metastasis as the first site of recurrence from cutaneous melanoma. J Eur Acad Dermatol Venereol. 2013;27(4):436-41.

4. Sartore L, Papanikolaou GE, Biancari F, Mazzoleni F. Prognostic factors of cutaneous melanoma in relation to metastasis at the sentinel lymph node: a casecontrolled study. Int J Surg. 2008;6(3):205-9.

5. Luo C, Shen J. Research progress in advanced melanoma. Cancer Lett. 2017;397:120-6.

6. Vichitvejpaisal P, Dalvin LA, Mazloumi M, Ewens KG, Ganguly A, Shields CL. Genetic Analysis of Uveal Melanoma in 658 Patients Using the Cancer Genome Atlas Classification of Uveal Melanoma as A, B, C, and D. Ophthalmology. 2019;126(10):1445-53.

7. Broekaert SM, Roy R, Okamoto I, van den Oord J, Bauer J, Garbe C, et al. Genetic and morphologic features for melanoma classification. Pigment Cell Melanoma Res. 2010;23(6):763-70.

8. Viros A, Fridlyand J, Bauer J, Lasithiotakis K, Garbe C, Pinkel D, et al. Improving melanoma classification by integrating genetic and morphologic features. PLoS Med. 2008;5(6):e120.

9. Azuma K, Sasada T, Takedatsu H, Shomura H, Koga M, Maeda Y, et al. Ran, a small GTPase gene, encodes cytotoxic T lymphocyte (CTL) epitopes capable of inducing HLA-A33-restricted and tumor-reactive CTLs in cancer patients. Clin Cancer Res. 2004;10(19):6695-702.

10. Sekiguchi T, Nishitani H, Nishimoto T. [The function of Ran GTPase cycle]. Tanpakushitsu Kakusan Koso. 1999;44(12 Suppl):1869-76.

11. Carey KL, Richards SA, Lounsbury KM, Macara IG. Evidence using a green fluorescent protein-glucocorticoid receptor chimera that the Ran/TC4 GTPase mediates an essential function independent of nuclear protein import. J Cell Biol. 1996;133(5):985-96.

12. Sazer S. The search for the primary function of the Ran GTPase continues. Trends Cell Biol. 1996;6(3):81-5.

13. Matchett KB, McFarlane S, Hamilton SE, Eltuhamy YS, Davidson MA, Murray JT, et al. Ran GTPase in nuclear envelope formation and cancer metastasis. Adv Exp Med Biol. 2014;773:323-51.

14. Li HY, Cao K, Zheng Y. Ran in the spindle checkpoint: a new function for a versatile GTPase. Trends Cell Biol. 2003;13(11):553-7.

15. Saxena S, Gandhi A, Lim PW, Relles D, Sarosiek K, Kang C, et al. RAN GTPase and Osteopontin in Pancreatic Cancer. Pancreat Disord Ther. 2013;3(1):113.

16. Sharma A, McCarron P, Matchett K, Hawthorne S, El-Tanani M. Anti-Invasive and Anti-Proliferative Effects of shRNA-Loaded Poly(Lactide-Co-Glycolide) 
Nanoparticles Following RAN Silencing in MDA-MB231 Breast Cancer Cells. Pharm Res. 2018;36(2):26.

17. Deng L, Lu Y, Zhao X, Sun Y, Shi Y, Fan H, et al. Ran GTPase protein promotes human pancreatic cancer proliferation by deregulating the expression of Survivin and cell cycle proteins. Biochem Biophys Res Commun. 2013;440(2):322-9. 18. Deng L, Shang Y, Guo S, Liu C, Zhou L, Sun Y, et al. Ran GTPase protein promotes metastasis and invasion in pancreatic cancer by deregulating the expression of AR and CXCR4. Cancer Biol Ther. 2014;15(8):1087-93.

19. Kurisetty VV, Johnston PG, Johnston N, Erwin P, Crowe P, Fernig DG, et al. RAN GTPase is an effector of the invasive/metastatic phenotype induced by osteopontin. Oncogene. 2008;27(57):7139-49.

20. Yuen HF, Chan KK, Grills C, Murray JT, Platt-Higgins A, Eldin OS, et al. Ran is a potential therapeutic target for cancer cells with molecular changes associated with activation of the PI3K/Akt/mTORC1 and Ras/MEK/ERK pathways. Clin Cancer Res. 2012;18(2):380-91.

21. Yuen HF, Chan KK, Platt-Higgins A, Dakir el H, Matchett KB, Haggag YA, et al. Ran GTPase promotes cancer progression via Met recepto-rmediated downstream signaling. Oncotarget. 2016;7(46):75854-64.

22. Haggag YA, Matchett KB, Dakir el H, Buchanan P, Osman MA, Elgizawy SA, et al. Nano-encapsulation of a novel anti-Ran-GTPase peptide for blockade of regulator of chromosome condensation 1 (RCC1) function in MDA-MB-231 breast cancer cells. Int J Pharm. 2017;521(1-2):40-53.

23. Doherty KJ, McKay C, Chan KK, El-Tanani MK. RAN GTPase as a target for cancer therapy: Ran binding proteins. Curr Mol Med. 2011;11(8):686-95.

24. Caenepeel S, Cooke K, Wadsworth S, Huang G, Robert L, Moreno BH, et al. MAPK pathway inhibition induces MET and GAB1 levels, priming BRAF mutant melanoma for rescue by hepatocyte growth factor. Oncotarget. 2017;8(11):17795809.

25. Elsheikh SE, Green AR, Rakha EA, Samaka RM, Ammar AA, Powe D, et al. Caveolin 1 and Caveolin 2 are associated with breast cancer basal-like and triplenegative immunophenotype. Br J Cancer. 2008;99(2):327-34.

26. Elsheikh SE, Green AR, Rakha EA, Powe DG, Ahmed RA, Collins HM, et al. Global histone modifications in breast cancer correlate with tumor phenotypes, prognostic factors, and patient outcome. Cancer Res. 2009;69(9):3802-9.

27. Fan H, Lu Y, Qin H, Zhou Y, Gu Y, Zhou J, et al. High Ran level is correlated with poor prognosis in patients with colorectal cancer. Int J Clin Oncol.

2013;18(5):856-63.

28. Caputo E, Wang E, Valentino A, Crispi S, De Giorgi V, Fico A, et al. Ran signaling in melanoma: implications for the development of alternative therapeutic strategies. Cancer Lett. 2015;357(1):286-96.

29. Bill KLJ, Garnett J, Ma XY, May CD, Bolshakov S, Lazar AJ, et al. The hepatocyte growth factor receptor as a potential therapeutic target for dedifferentiated liposarcoma. Lab Invest. 2015;95(8):951-61.

Figure 1: Ran protein expression in malignant melanoma using immunohistochemistry: $A, B, C$, and $D$ represent Ran positive melanoma cases $(\mathrm{H}-$ 
score >102). E and F Ran negative case $(H$-score <102). A, C, and E original magnification X50, B, D and F original magnification X400.

Figure 2: Kaplan-Meier plots for Ran immunohistochemical expression in malignant melanoma. (A) Lymph node metastasis-free survival and (B) Overall survival.

Figure 3: Western blot for total Ran, Met, ERK1/2, phospho-ERK1/2 and a-tubulin for A375 and G361 cell lines transfected with 120nM of siLuc, siRAN1 or siRAN2.

Figure 4: Ran knockdown reduces melanoma cell migration in A375 and G361 cell lines (A) and in A375 measured via wound healing assay as shown in a snapshot of some of the reduplicate groups (B). Ran knockdown reduces melanoma cell invasion in both cell lines (C). Bars represent standard deviation of the mean (SDM). Cl: $95 \%$. ${ }^{\star} \mathrm{P}<0.05,{ }^{* *} \mathrm{P}<0.01,{ }^{* * *} \mathrm{P}<0.001,{ }^{* * * *} \mathrm{P}<0.0001$. Technical repeats for each assay $=$ 3. Measurements were taken at $24 \mathrm{~h}$ of incubation.

Figure 5: Ran knockdown reduces cell proliferation in (A) A375 and (B) G361 cell lines. RFU: relevant fluorescence units. Bars represent standard deviation of the mean (SEM).Cl: 95\%. Technical repeats $=12 .{ }^{*} \mathrm{P}<0.001$

\section{Figure 6:}


Figure 1

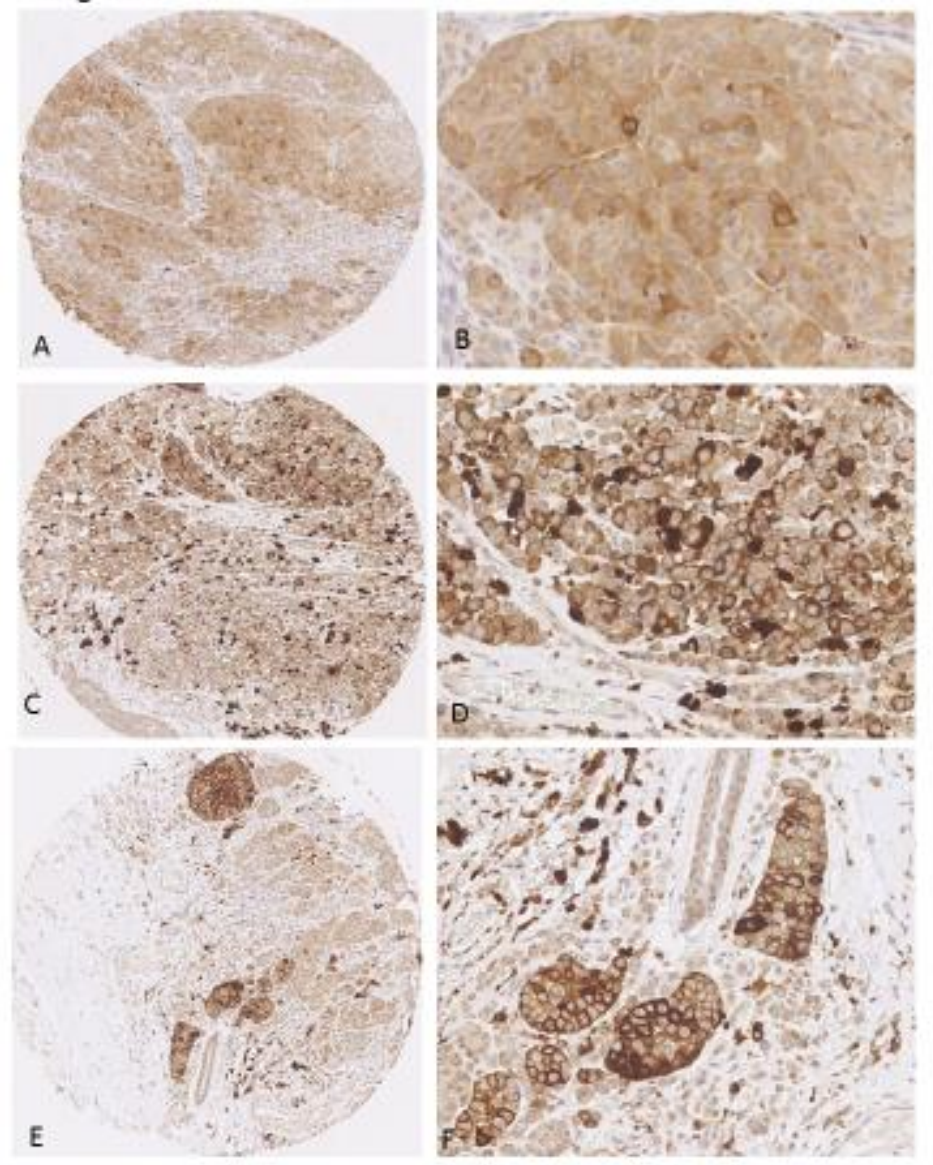


Figure 2
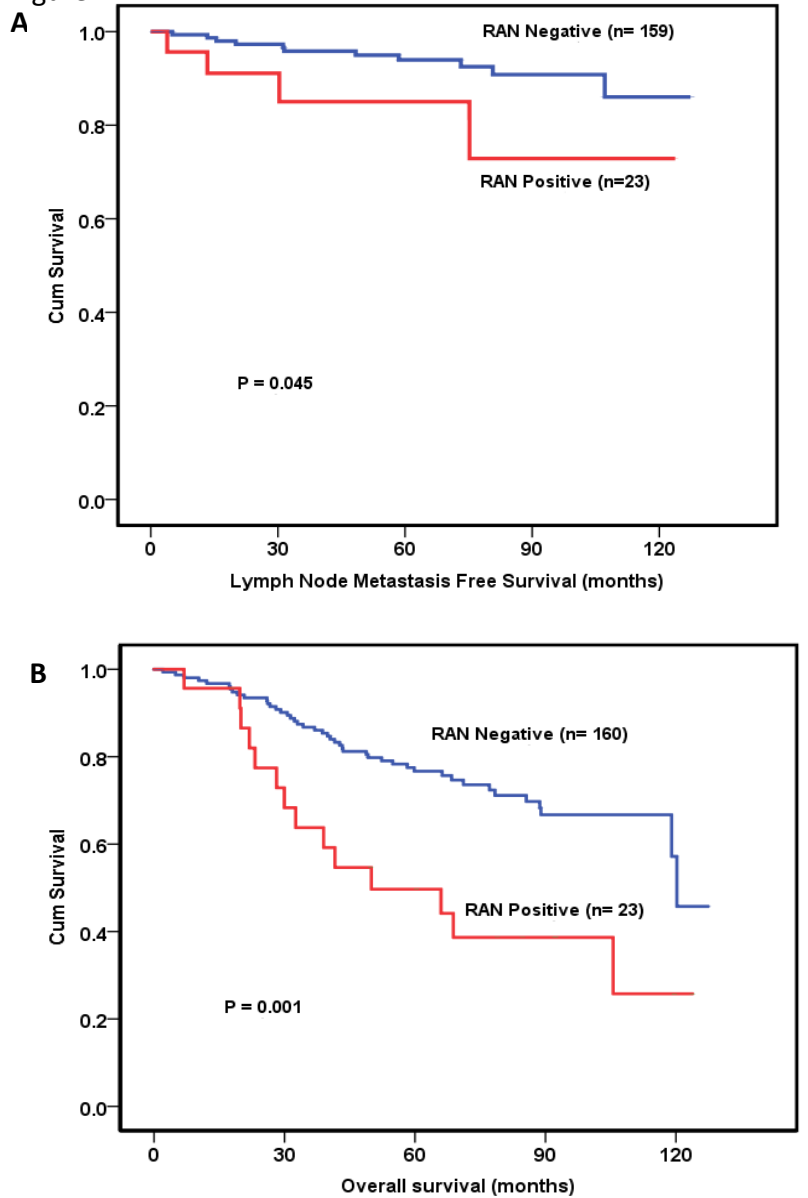

Figure 3

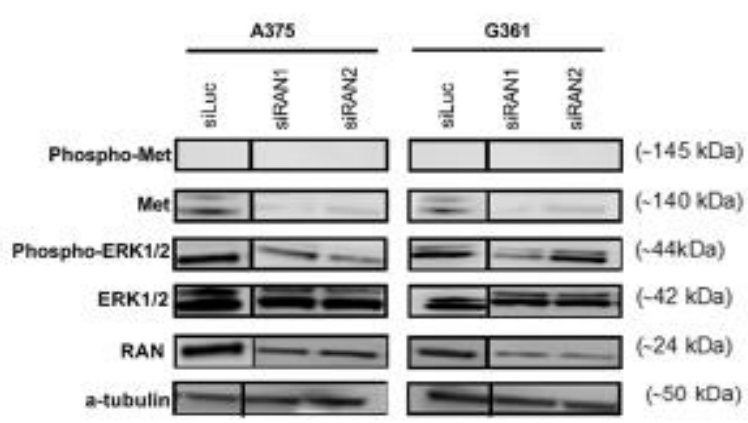


A Figure 4
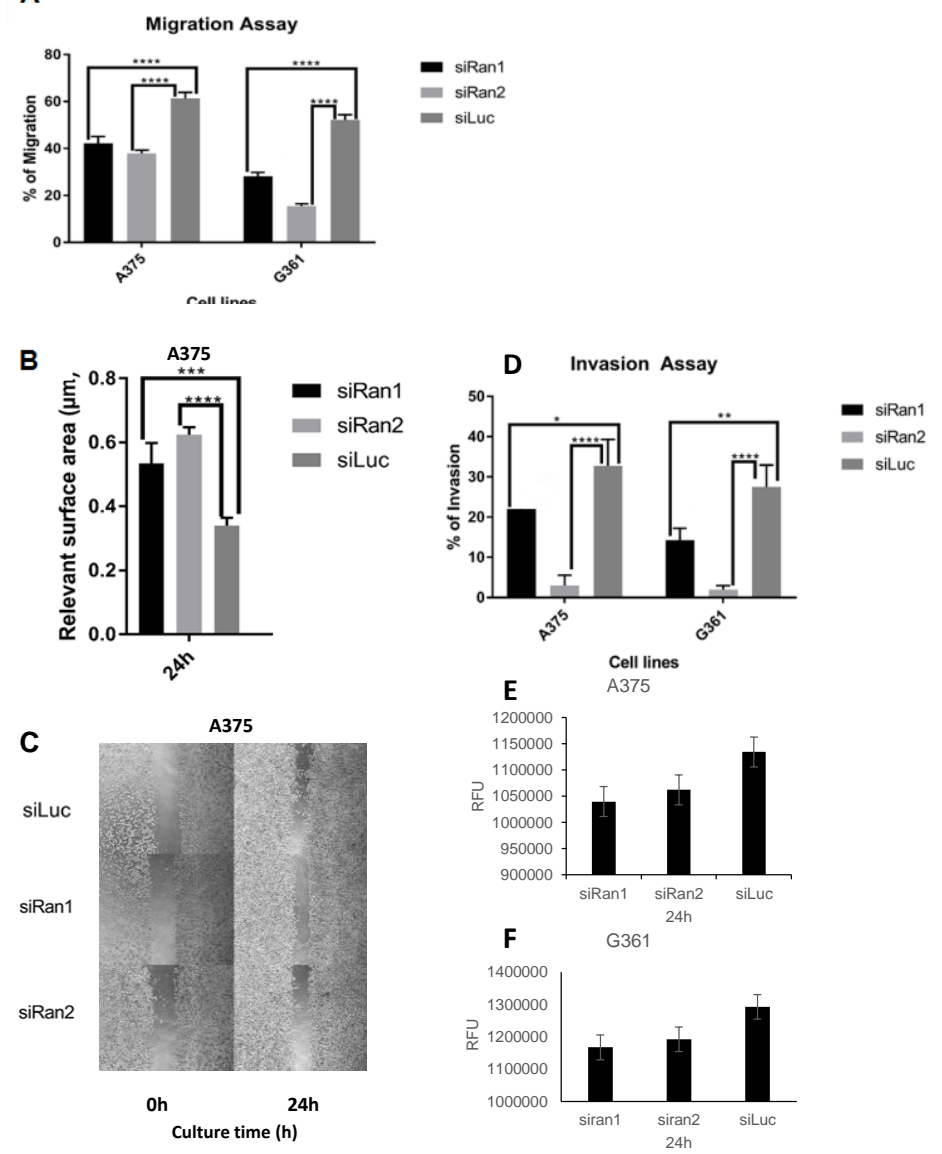


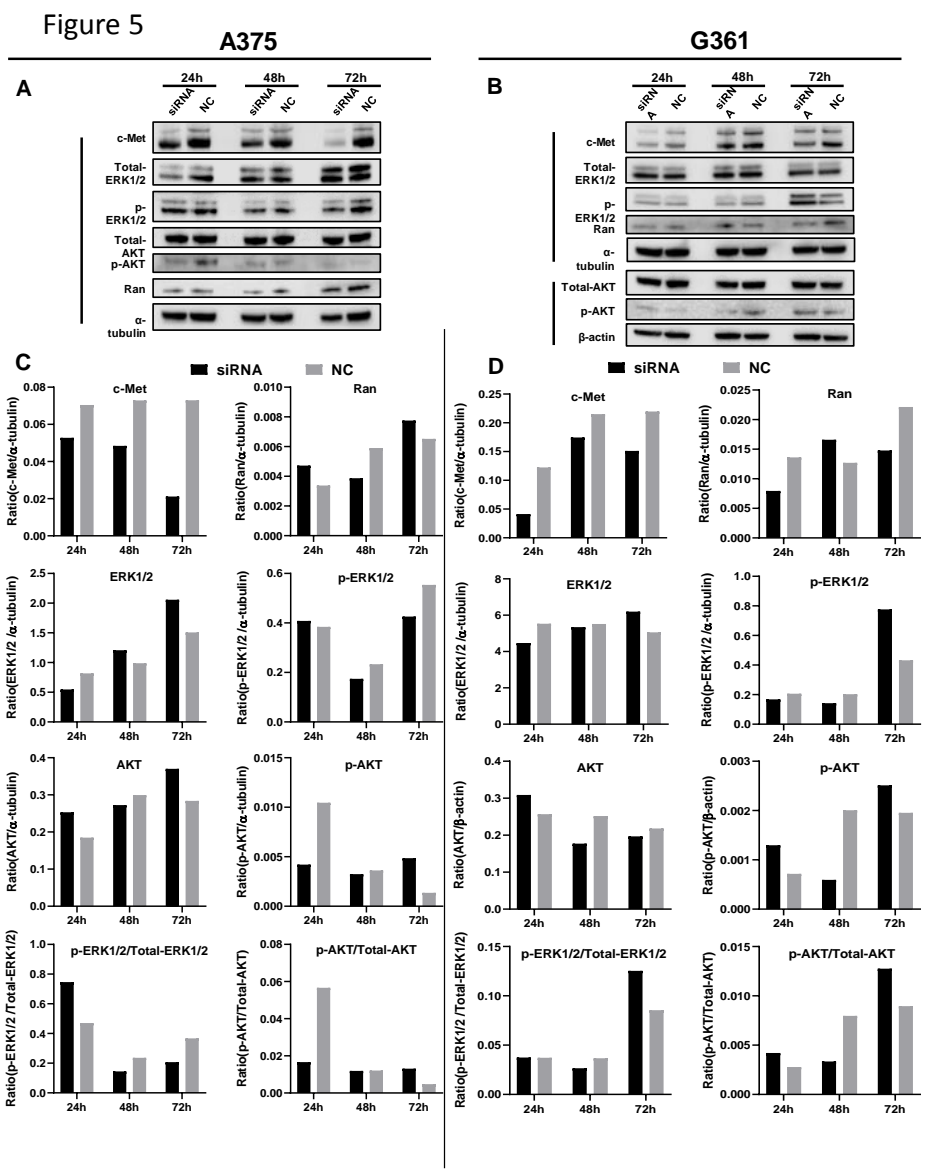



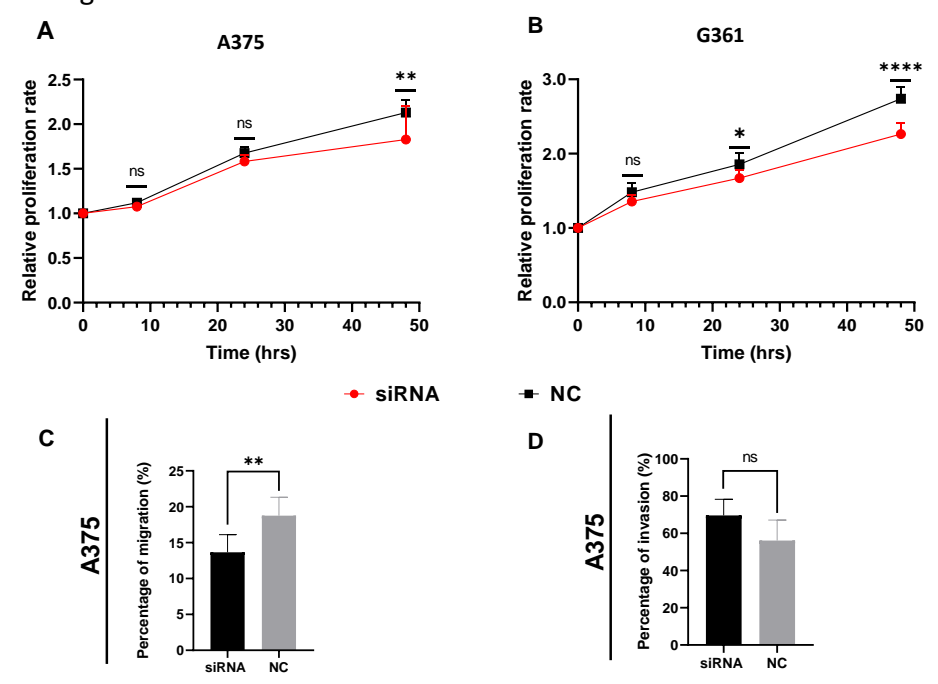

- NC

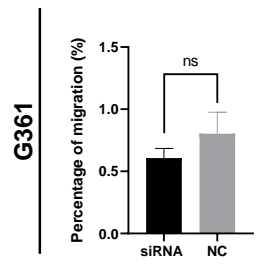

D
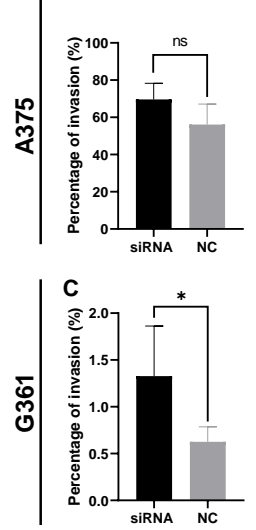

\section{Supplemental Material and methods}

\section{Clinicopathological data}

Tumours were surgically excised, formalin-fixed and paraffin embedded (FFPE) in tissue blocks. The main selection criterion was tumours having a Breslow thickness of $>1 \mathrm{~mm}$. This selection should allow enough material to assess Ran protein expression at a substantial amount of invasive melanoma. Patients gave informed consent for their specimens to be, harvested, stored and used for research. Data and specimens were anonymised by using only a unique anonymisation code. Ethical approval (ACP0000174) was gained from the Nottingham Health Science Biobank Access Committee. A cohort of 228 primary MM cases was used for immunohistochemistry staining in addition to 20 cases with full-face sections. Patient clinicopathological data was obtained from the Nottingham University Hospitals patients' information databases.

Sixty-three cases from the initial cohort of 248 were excluded due to tissue loss or insufficient melanoma cells present for scoring. 
In this cohort, there were slightly more males (53\%) than females (47\%), and most patients aged 50-70 at diagnosis, with a mean age of 63.5 years. Superficial spreading malignant melanoma subtype represents $44.9 \%$ of all cases. The majority of melanoma cases had a Breslow thickness of $>1-2 \mathrm{~mm}(38.4 \%)$ with no ulceration (73.5\%), no microsatellites (97.8\%), and mitosis was non-brisk [mitosis $<4$ per mm2] (64.7\%). Only $4.3 \%$ of cases had local recurrence and $23.3 \%$ developed metastatic disease (lymph node or distant metastasis), with the mean metastasis-free survival being 54.4 months ( $\mathrm{SE} \pm 1.9$ months). The majority of patients were alive when study data was collected, with only $13 \%$ of cases died due to melanoma. The mean survival time was 94.8 months (SE \pm 3.4 months).

\section{Tissue microarray (TMA) construction}

TMA blocks were constructed using the TMA Grand Master system (3DHistech, Budapest, Hungary). Target areas were selected on haematoxylin and eosin-stained sections from representative FFPE blocks for each case. The location of cores to sample from the tissue block were annotated on scanned images of the sections using Pannoramic Viewer software (3DHistech). Cores were removed from the FFPE tissue blocks using the TMA Grand Master (3DHisTech) and arrayed into the recipient paraffin blocks. Three cores were selected from each target tumour area and a total of 228 cases were arrayed onto the TMA.

\section{Immunohistochemistry staining and tissue scoring}

Tissue sections, $3 \mu \mathrm{m}$ thick, were cut from patient specimen FFPE full-face tissue blocks and TMA blocks. Slides were baked at $60^{\circ} \mathrm{C}$ for 1 hour to ensure tissue adherence prior to immunohistochemical staining using UltraView Universal DAB detection kit (Roche Ventana \#05269806001) on BenchMark ULTRA automated IHC/ISH slide staining system (Roche Ventana) which utilises pre-diluted Liquid Coverslip (LCS) (Roche Ventana \#05424534001) to prevent drying out of tissues throughout the staining procedure. All steps were performed at $21^{\circ} \mathrm{C}$, unless stated otherwise. Briefly, slides were dewaxed at $72^{\circ} \mathrm{C}$ for 12 minutes in EZ Prep (Roche Ventana \#05279771001) before antigen retrieval was performed using pre-diluted Tris-based buffer Cell Conditioning Solution 1 (pH8) (Roche Ventana \#05279801001) for 64 minutes, followed by a wash step using Reaction Buffer (Roche Ventana \#05353955001). Endogenous peroxidase activity was blocked using $3 \% \mathrm{H}_{2} \mathrm{O}_{2}$ solution (UltraView Detection kit). Rabbit Anti-Ran polyclonal antibody (Abcam, ab4781) diluted in Antibody Diluent (Roche Ventana number 251- 
018) at 1:100 was then applied to each slide and incubated for 32 minutes, followed by another wash step. Slides were then incubated with a Horseradish Peroxidase multimer (UltraView Detection kit) for 8 minutes, for signal amplification, followed by another wash step. The DAB chromogen (0.2\%) and peroxidase enzyme $(0.04 \%$ $\mathrm{H}_{2} \mathrm{O}_{2}$ in PBS) from the UltraView Detection kit, were then mixed, applied to the slides and incubated for 8 minutes, followed by another wash step. The slides were then incubated with copper sulphate (5g/L) (UltraView Detection kit) for 4 minutes, for stain enhancement, followed by another wash step. Sections were then counterstained with Haematoxylin II (Roche Ventana \#05277965001) for 12 minutes. Slides were rinsed with EZ Prep to remove residual LCS, dehydrated with IMS, cleared with xylene, and permanently mounted in pertex under a glass coverslip. Breast carcinoma tissue has been used as a positive control. Slides were scanned on the Nanozoomer C9600-01 (NanoZoomer; Hamamatsu Photonics, Welwyn Garden City, UK) and images were edited/annotated using NDP.view 2 software version 2.6.13. Scoring was done using the $\mathrm{H}$-score method.

\section{Statistical analysis}

Data sets were statistically compared and analysed by two-way factorial ANOVA and post-hoc (Sidak). The ImageJ software (NIH, USA) was used to quantify the wound healing assay results as follows: File > Open Image, Image > Type > 8-bit then Adjust $>$ Threshold $>$ reset, Process $>$ FFT $>$ Bandpass filter $>$ OK, Image $>$ Adjust $>$ Threshold $>$ set, Process $>$ Filters $>$ Minimum $>$ Set Radius at 7.0, Wand tool $>$ select wound healing surface area, Analyse > Measure. IBM SPSS software version 23 (Chicago, IL, USA) was used to analyse the relation between Ran protein expression in relation to clinicopathological parameters and survival. Kappa coefficient $(\mathrm{K})$ test was used to measure inter-observer agreement. The association of Ran expression with clinicopathological parameters was assessed using the chisquared test or Fisher's Exact Test. Ran expression with metastasis-free survival and overall survival was assessed using the Kaplan-Meier Log Rank test. Cox hazard regression analysis was performed to quantify the risk of clinicopathological parameters and Ran expression on survival. A P-value of $<0.05$ (two-tailed) was considered to denote statistical significance.

Multiple clinicopathological parameters are associated with lymph node metastasis-free survival and overall survival in this cohort of patients. 
Kaplan-Meier Log Rank tests were used to assess the relation of clinicopathological parameters with lymph node metastasis-free survival and overall survival in this cohort of patient's Supplementary table 2. Lymph node metastasis-free survival was defined as the duration (in months) from the date of primary melanoma diagnosis to the date of lymph node metastasis, while overall survival was defined as the duration (in months) from the date of primary MM to the date of death caused by melanoma. As expected, Breslow thickness was a significant prognostic factor, being highly significantly associated with overall survival $(p<0.001)$ and significantly associated with lymph node metastasis free survival $(p=0.038)$. Gender $(p=0.006)$, age $(p<0.001)$, ulceration $(p<0.001)$ and mitosis $(p=0.012)$ were also significantly associated with overall survival. Whereas microsatellites was not significantly associated $(p>0.05)$.

\section{Supplementary Table 1S: Summary of the clinicopathological data for}

\section{melanoma patients}

\begin{tabular}{|c|c|c|}
\hline \multicolumn{2}{|l|}{ Factor } & \multirow{2}{*}{$\begin{array}{l}\text { Frequency n (\%) } \\
98(53)\end{array}$} \\
\hline Gender & Male & \\
\hline & Female & $87(47)$ \\
\hline Age at primary & $<50$ & $36(19.5)$ \\
\hline \multirow[t]{2}{*}{ diagnosis (years) } & $50-70$ & $83(44.9)$ \\
\hline & $>70$ & $66(35,7)$ \\
\hline \multirow[t]{5}{*}{ Histological Subtype@ } & Unknown & $53(28.6)$ \\
\hline & SSMM & $83(44.9)$ \\
\hline & NM & $30(16.3)$ \\
\hline & ALM & $8(4.3)$ \\
\hline & LMM & $11(5.9)$ \\
\hline Thickness & $T 2>1-2$ & $71(38.4)$ \\
\hline \multirow[t]{2}{*}{$(\mathrm{mm})$} & $T 3>2-4$ & $65(35,1)$ \\
\hline & $\mathrm{T} 4>4$ & $49(26,5)$ \\
\hline \multirow[t]{4}{*}{ Clinical Stage } & IA & $2(1.1)$ \\
\hline & IB & $62(33.5)$ \\
\hline & IIA & $38(20.5)$ \\
\hline & IIB & $28(15.1)$ \\
\hline
\end{tabular}




\begin{tabular}{|l|l|l|l} 
& IIC & $20(10.8)$ \\
& III & $2(1.1)$ \\
& IIC & $9(4.9)$ \\
& IV & $24(13.0)$ \\
\hline Ulceration & Absent & $136(73.5)$ \\
& Present & $49(26,5)$ \\
\hline Microsatellites & Absent & $180(97,8)$ \\
\hline Mitosis & Present & $4(2.2)$ \\
& Absent & $10(5.4)$ \\
& Non-brisk $\left(<4 / \mathrm{mm}^{2}\right)$ & $119(64.7)$ \\
& Brisk $\left(>4 / \mathrm{mm}^{2}\right)$ & $55(29.9)$ \\
\hline Local Recurrence & Absent & $177(95.7)$ \\
& Present & $8(4.3)$ \\
\hline Lymph & Absent & $170(91.9)$ \\
\hline Metastasis & Present & $15(8.1)$ \\
& & \\
\hline Distant Metastasis & Absent & $157(84,9)$ \\
& Present & $28(15.1)$ \\
\hline Mortality & Alive & $127(68.6)$ \\
& Death due to melanoma & $34(13)$ \\
& Death due to other & $34(18.4)$ \\
& causes & \\
\hline
\end{tabular}

(c) Main Histological subtypes are SSMM: superficial spreading malignant melanoma. NM: Nodular melanoma. ALM: Acral lentiginous melanoma. LMM: Lentigo maligna melanoma.

Supplementary Table 2S: Association of Ran expression with clinicopathological parameters in malignant melanoma cases. 


\begin{tabular}{|c|c|c|c|c|}
\hline Factor & Number & $\begin{array}{l}\text { Ran negative } \\
(\leq 102) \text { N (\%) }\end{array}$ & $\begin{array}{l}\text { Ran positive } \\
(>102) \mathrm{N}(\%)\end{array}$ & $p$-value \\
\hline Gender & 185 & & & $0.506^{\star \star \star}$ \\
\hline Male & 98 & $84(51.9)$ & $14(60.9)$ & \\
\hline Female & 87 & $78(48.1)$ & $9(39.1)$ & \\
\hline $\begin{array}{l}\text { Age at primary } \\
\text { diagnosis (years) }\end{array}$ & 185 & & & $0.004^{\star}$ \\
\hline$<50$ & 71 & $30(18.5)$ & 63 (26.1) & \\
\hline $50-70$ & 65 & $80 \quad(49.4)$ & $3(13.0)$ & \\
\hline$>70$ & 49 & $52 \quad(32.1)$ & $14(60,9)$ & \\
\hline Histological subtype & 185 & & & $0.323^{*}$ \\
\hline SSM & 83 & 75 (46.3) & $8(34.8)$ & \\
\hline NM & 30 & 27 (16.7) & $3(13)$ & \\
\hline$\overline{A L M}$ & 8 & $6(3.7)$ & $2(8.7)$ & \\
\hline LMM & 11 & $11(6.8)$ & $0(0)$ & \\
\hline Unknown & 53 & $43(26.5)$ & $10(43.5)$ & \\
\hline $\begin{array}{l}\text { Breslow Thickness } \\
(\mathrm{mm})\end{array}$ & 197 & & & $0.019^{\star \star \star}$ \\
\hline$T 2>1-2$ & 71 & $68(42.0)$ & $3(13.0)$ & \\
\hline$T 3>2-4$ & 65 & $52(32.1)$ & $13(56.5)$ & \\
\hline $\mathrm{T} 4>4$ & 49 & $42(25,9)$ & $7(30.4)$ & \\
\hline Clinical Stage & 185 & & & $0.351^{*}$ \\
\hline IA & 2 & $2(1.2)$ & $0(0)$ & \\
\hline IB & 62 & $59(36.4)$ & $3(13)$ & \\
\hline IIA & 38 & $33(20.4)$ & $5(21.7)$ & \\
\hline$\overline{I I B}$ & 28 & $22(13.6)$ & $6(26.1$ & \\
\hline IIC & 20 & $16(9.9)$ & $4(17.4)$ & \\
\hline III & 2 & $2(1.2)$ & $0(0)$ & \\
\hline IIIC & 9 & $7(4.3)$ & $2(8.7)$ & \\
\hline
\end{tabular}




\begin{tabular}{|l|l|l|l|l|}
\hline IV & 27 & $21(13 \%)$ & $8(13 \%)$ & \\
\hline Ulceration & 185 & & & $0.204^{\star \star \star}$ \\
\hline Absent & 136 & $122(75.3 \%)$ & $40(60.9 \%)$ & \\
\hline Present & 49 & $14(24.7 \%)$ & $9(39.1 \%)$ & \\
\hline Microsatellites & 184 & & & $0.583^{\star \star \star}$ \\
\hline Absent & 180 & $157(97.5 \%)$ & $4(100.0 \%)$ & \\
\hline Present & 4 & $4(2.5 \%)$ & $0(0 \%)$ & \\
\hline Mitosis & 184 & & & $0.062^{\star}$ \\
\hline Absent & 10 & $10(6.2 \%)$ & $0(0.0 \%)$ & \\
\hline Non-brisk $\left(<4 / \mathrm{mm}^{2}\right)$ & 119 & $188(66.7 \%)$ & $11(50.0 \%)$ & \\
\hline Brisk $\left(>4 / \mathrm{mm}^{2}\right)$ & 55 & $44(27.2 \%)$ & $11(50.0 \%)$ & \\
\hline Local Recurrence & 185 & & & 0.276 \\
\hline Absent & 177 & $154(95.1 \%)$ & $23(100.0 \%)$ & \\
\hline Present & 8 & $8(4.9 \%)$ & $0(0.0 \%)$ & \\
\hline
\end{tabular}

${ }^{*}$ Chi-squared test, ${ }^{* *}$ Chi-squared test for trend, ${ }^{* * *}$ Fisher's exact test.

\section{Cell culture}

Melanoma cell lines, A375 and G361 (ECACC) derived from patients (Life Technologies, 41965-039) were cultured in DMEM supplemented with $10 \%(\mathrm{~m} / \mathrm{v})$ foetal bovine serum. When cells were near confluence, the media were aspirated off and cells were gently washed with PBS and incubated in $5 \% \mathrm{CO}_{2}$ at $37^{\circ} \mathrm{C}$ with Trypsin/EDTA solution for 5 minutes. Following incubation, fresh media were added and cells were transferred in sterile universal tubes and centrifuged at $300 \times \mathrm{g}$ for 5 minutes. Supernatants were aspirated off and cell pellets were re-suspended in new media. Fresh media were then added to sterile T75 flasks before adding proportions of re-suspended cells. An automated cell counter was used to count cells. Differ Small interfering RNA transfection.

\section{Small interfering RNA transfection}


The two independent RAN siRNAs (siRAN1 Sense 5' G U U U G A U G U A A C A U CGAGAUU-3' ; Antisense 5' -AAUCUCGAUGUUACAUCAAAC-3' ; siRAN2 Sense 5'CACCAACAGAGGACCUAUUAA-3';Antisense5'-UUAAUAGGUCCUCUGUUGGUG3') and the control siRNA (siLuc) were purchased from Eurofins Genomics. Cells were seeded (3x 105 cells/ well) on 6-well culture dishes and were allowed to grow to approximately $70 \%$ confluence. On the day of transfection, normal DMEM were removed and $2 \mathrm{ml}$ of reduced serum medium (Opti-MEM, Life Technologies, 31985047) were added to each well. The wells were incubated in $5 \% \mathrm{CO}_{2}$ at $37^{\circ} \mathrm{C}$ for 60 minutes. Meanwhile, the transfection complexes were prepared. In separate Eppendorf tubes, 100 $\mu$ Mof each RAN siRNA (siRAN1, siRAN2 and siLuc) were diluted in $250 \mu \mathrm{l}$ of Opti-MEM. For each of the siRNAs, $5 \mu \mathrm{l}$ of Lipofectamine 2000 reagent (Invitrogen, 11668-19) were diluted in 250 $\mu$ l of Opti-MEMin separate tubes. The solutions were incubated briefly for 5 minutes at room temperature. Then, each siRNA solution was gently mixed with Lipofectamine and the complexes were incubated for an additional 20 minutes at room temperature. Transfection complexes were added to each well and incubated for 6 hours. Opti-MEM were removed and replaced with DMEM after the incubation period. Cells were harvested 24 hours and 48 hours posttransfection for functional assays and protein extractions respectively. Results were compared against cells transfected with a siRNA that targeted the luciferase gene (control). Differentiation between live and dead cells was determined based on trypan blue.

Using two separate Ran siRNAs at different concentrations, Ran knockdown was confirmed with WB and densitometry (Sup. Fig. 2) analyses. In A375 cells, 40 nM, 80 $\mathrm{nM}$ and $120 \mathrm{nM}$ of siRan1 resulted in $83 \%, 85 \%$ and $94 \%$ knockdown efficiencies and the same concentrations of siRan2 gave efficiencies of $86 \%, 84 \%$ and $90 \%$ respectively. In G361 cells, knockdown efficiencies were approximately 76\%, 78\% and $80 \%$ for siRan 1 and $78 \%, 79 \%$ and $85 \%$ for siRan2. To evaluate the functional relevance of both Ran siRNAs, results from Ran siRNA-transfected cells were normalised against those from luciferase siRNA-transfected cells.

\section{Gradient and real-time polymerase chain reaction}

Total RNA was extracted from cells using the GenEluteMiniprep Kit (Sigma-Aldrich, RTN70-1KT) and DNase treatment was performed using the on-column DNase Digestion Set (Sigma-Aldrich, DNASE70-1SET) according to the manufacturer's instructions. Extracted RNA was quantified using NanoDrop2000 (Thermo Fisher 
Scientific). Before reverse transcription, a master mix was prepared for each reaction $(2.5 \mu \mathrm{l}$ of random hexamers were added to $2 \mu \mathrm{g}$ of total RNA and made up to a total volume of $15 \mu \mathrm{l}$ with $\mathrm{dH}_{2} \mathrm{O}$ ) and incubated at $70^{\circ} \mathrm{C}$ for 5 minutes and then on ice for 5 minutes. To every master mix, $5 \mu$ l of $5 x$ buffer, $1.25 \mu$ l of dNTPs, $1 \mu l$ of M-MLV enzyme, $0.625 \mu \mathrm{l}$ of RNAse inhibitor (all Promega, UK) and $2.125 \mu \mathrm{l}$ of $\mathrm{dH}_{2} \mathrm{O}_{\text {were }}$ added (final volume $25 \mu \mathrm{l}$ ). The reactions were incubated at $37^{\circ} \mathrm{C}$ for 60 minutes and $95^{\circ} \mathrm{C}$ for 10 minutes.

Primers for the RAN gene (Forward 5'-GCACGACTTAGAGGTTGCTCA-3'; Reverse 5'-GCTTCATTCTCACAGGTCATCAT-3') were designed using OligoAnalyzer software (Integrated DNA Technologies). Additionally, pre-designed primers were purchased (Eurofins). Ribosomal protein S23 (RPS23) was used as an internal control (Forward 5'-TAGTCACCGACGAGACCAGA-3'; Reverse 5'-TCAACTCCTAC TTTTTCCAGCAC-3'). Gradient PCR was performed for primer annealing temperature optimisation. Then, the gradient PCR product was analysed upon LightScanner (Idaho Technology, Inc.) using high resolution melting. Real-time PCR was performed using GoTaqMastermix (Promega, UK, A600A). Reactions were analysed upon Stratagene Mx3005P using the following cycle conditions: $50^{\circ} \mathrm{C}$ for 10 minutes, $95^{\circ} \mathrm{C}$ for 2 minutes, followed by 40 cycles at $95^{\circ} \mathrm{C}$ for 30 seconds and $60^{\circ} \mathrm{C}$ for 1 minute. The results from qPCR were normalised against RPS23 expression using the $\Delta \Delta \mathrm{Ct}$ method.

\section{Western blot analysis}

Cells were lysed in lysis buffer (Thermo Fisher Scientific, 87787) containing a protease inhibitor cocktail (Thermo Fisher Scientific, 1861281) for 15 minutes at $4^{\circ} \mathrm{C}$ with constant agitation. Protein amounts were quantified using the BCA assay kit (Thermo Fisher Scientific, 23225). The respective protein amounts were separated using SDSPAGE $4-12 \%$ NuPAGE Gels (Thermo Fisher Scientific, NP0335BOX) and transferred to PVDF membrane (GE Healthcare, 10600023). The proteins of interest were detected with specific primary antibodies: Rabbit Ran (diluted 1:1000, Millipore,07517) and mouse a-tubulin (diluted 1:2000, Abcam, DM1A). Further, Rabbit Met mAb (diluted 1:1000, D1C2), Rabbit Phospho-Met mAb (diluted 1:1000, D26), Rabbit ERK1/2 mAb (diluted 1:1000, 137F5) and Rabbit Phospho-ERK1/2 mAb (diluted 1:2000, D13.14.4E) were used (all purchased from Cell Signalling). After three further washes, detection was performed using the Enhanced Chemiluminescence Kit (Pierce, Thermo Fisher, UK). Bands were visualised using C-digit scanner (Li-Cor) and quantified by densitometry using Studio Lite (Li-Cor). 


\section{PrestoBlue cell viability assay}

The seeding densities of cell lines were first optimised. Cells $\left(10^{5} \mathrm{celll} /\right.$ well) were seeded on 96-well culture dishes and incubated overnight to allow cells to adhere. The following day PrestoBlue (Life Technologies, A13261) was diluted 1:10 in culture media and incubated at room temperature for 10 minutes. The media were removed from the wells and replaced with aliquots of $100 \mu \mathrm{IPrestoBlue}$ solution and the plates were incubated for 60 minutes at $37^{\circ} \mathrm{C}$. Plates were analysed upon FlexStation II (Molecular Devices, USA) and fluorescence values were obtained (Excitation/Emission: $540 \mathrm{~nm} / 610 \mathrm{~nm}$, bottom reads). Sterile DMEM were used as negative controls.

\section{Transwell migration and invasion assay}

Approximately $10^{4}$ cells in serum-free conditions were seeded into the top of each transwell (Corning, 3422) with or without Matrigel Matrix coating (Corning, 356230) for invasion or migration assays respectively. The cells were allowed to migrate or invade towards the bottom of the transwell with $10 \%$ FBS as a chemoattractant for 24 hours incubated as normal. Then, all the transwells were moved to empty wells where trypsin was added to harvest the cells that migrated/invaded the bottom of the transwell. The trypsinised media were then transferred to the previous wells as appropriate and $1 \mathrm{ml}$ of DMEM was added to every well to ensure trypsinisation had stopped. Cells were stained with Calcein AM (eBioscience, 65-0853-39) and incubated as normal for 30 minutes. Receiver wells with no chemoattractant were used as controls. Assays were performed in triplicate and on at least two separate occasions.

After $24 \mathrm{~h}$ of transfection with the siRNA, the cells were digest with trypsin, washed and counted. Five $\times 104$ cells were seeded in serum-free DMEM in the top chamber and allowed to migrate towards the lower chamber containing $10 \% \mathrm{v} / \mathrm{v}$ FBS as a chemoattractant (complete growth medium). After 24 hours, migration was assessed as follows: transwells were moved to empty wells where trypsin was added to harvest the cells that were present on the underside of the membrane. The trypsinised cells and media were then transferred to the original wells and $1 \mathrm{ml}$ of complete growth medium DMEM was added to every well to ensure that trypsinisation had stopped. Viable cells were stained by incubating with $2 \mu \mathrm{M}$ Calcein AM (eBioscience) for 30 minutes and then counted. Receiver wells with no chemoattractant were used as controls. Migration percent was defined as the mean count of cells migrated with chemoattractant divided by the mean count of cells seeded $x 100$. 


\section{Wound healing assay}

Wound healing assays were performed in 24-well culture dishes. Culture inserts (Ibidi, 80209) were firmly attached to each well and $0.5 \times 105$ cells were added into each well. The plates were incubated overnight and checked to confirm confluence, the inserts were removed, cells were washed with sterile PBS and then DMEM containing $10 \%$ FBS was added. Cells were imaged at 0 and 24 hours using Nikon Eclipse Ti2 microscope. Results were analysed using the Image J software (NIH, USA).

\section{Cell viability assay}

A resazurin-reduction assay was performed by diluting PrestoBlue (Life Technologies, A13261) at $10 \%$ in complete cell growth medium (DMEM, 10\%FBS, $4 \mathrm{mM}$ LGlutamine). The plates were incubated for 60 minutes at $37 \mathrm{oC}$ and then bottom-read at 540nm Excitation /610nm Emission filters on the FlexStation II (Molecular Devices) fluorescence plate reader. The fluorescence values of blank wells were subtracted from the raw values before normalisation to the first time point. 

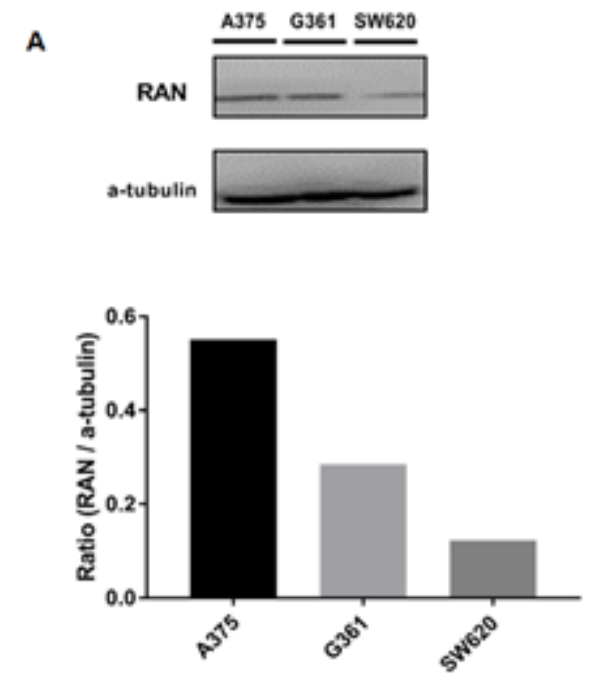

B

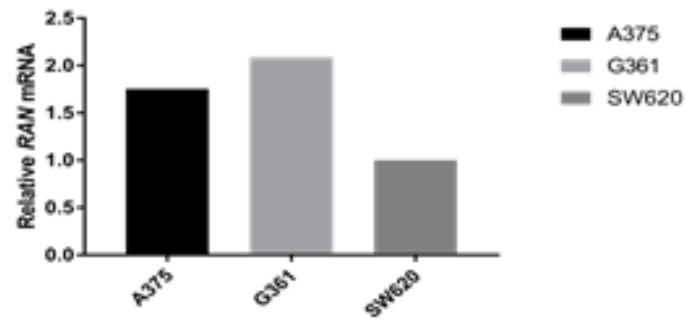

Sup Figure 1

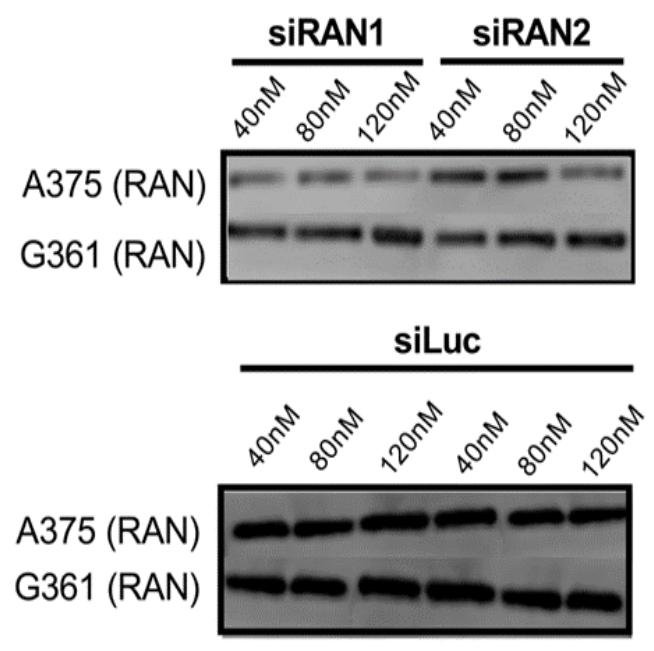




\section{Sup Figure 2}

\section{Supplementary Figure Legend}

Supplementary Figure 1: Ran expression on transcriptional (mRNA) and posttranscriptional (protein) levels in malignant melanoma (A375, G361) and colorectal cancer (SW620) cell lines. A: Western blot for Ran and a-tubulin (control) and densitometry analysis. B: qPCR for Ran expression normalised against RPS23 (control) expression. Reactions in qPCR were performed in triplicates.

Supplementary Figure 2: Optimisation of Ran siRNA concentrations with the respective densitometry analyses. 